\title{
EL MÉTODO DE AUTO-CONFRONTACIÓN: EVALUACIÓN, CAMBIO Y VALORACIÓN DE LOS SISTEMAS DE SIGNIFICADO PERSONAL
}

\author{
Hubert J. M. Hermans \\ University of Nijmegen \\ The Netherlands
}

\begin{abstract}
RESUMEN
El método de auto-confrontación, en un intento de unir la brecha existente en psicologia entre científicos y profesionales, se diseña, tanto como instrumento de investigación, como procedimiento práctico para ayudar a los clientes en su autoinvestigación. Se describirá un caso en detalle para demostrar las tres funciones del método: (a) como un método de evaluación para la investigación del contenido y estructura del sistema de valoración; (b) como una herramienta para la reorganización de este sistema; y (c) como un instrumento para la valoración de la naturaleza del cambio del sistema. Para finalizar, se resumirán algunas áreas problemáticas y campos de aplicación en los cuales se ha empleado dicho método.
\end{abstract}

Palabras clave: si-mismo, auto-confrontación, auto-investigación, significados personales, teoria de la valoración, psicoterapia.

\begin{abstract}
As an attempt to bridge the scientist-practitioner split in psychology, the selfconfrontation method is deviced as both a research instrument and practical procedure for assinsting clients in their self-investigation. A case study is described in detail in order to demonstrate the three functions of the method: (a) as an assessment method for the investigation of the content and structure of the valuation system: (b) as a tool for the reorganization of this system: and (c) as an instrument for the evaluation of the nature of the change of the system. Finally, some problem aras and fields of application in which the method has been applied, are summarized.
\end{abstract}

Key words: self, self-confrontation, self-investigation, personal meanings, valuation theory, psychotherapy.

Correspondencia: Hubert J. M. Hermans, Department of Clinical Psychology and Personality, University of Nijmegen, P.O. Box 9104, 6500 HE Nijmejen, The Netherlands. E-mail: hhermans@psych.kun.nl 
El método de auto-confrontación es una aproximación individualizada a las auto-narrativas basada en la teoria de valoración, que puede ser utilizado para tres propósitos: evaluación, facilitación del proceso, y valoración del sistema de significados de la persona. Es, además, un instrumento útil tanto para la investigación como para la práctica clínica.

Este capitulo contiene (a) una descripción del método en si mismo, con especial atención a su evaluación, a la facilitación del proceso, y a sus fines de valoración; (b) una ilustración con un caso real; y (c) una visión globalizadora de sus aplicaciones. Hermans y Hermans-Jansen (1995) presentan un tratamiento comprehensivo y teóricamente fundamentado del método, en combinación con diversos estudios de caso clínicos.

\section{EL MÉTODO DE AUTO-CONFRONTACIÓN: LAS VALORACIONES Y SUS PROPIEDADES AFECTIVAS}

El método de auto-confrontación invita a una persona (sujeto o cliente) a llevar a cabo una minuciosa auto-investigación consistente en tres partes: (a) la construcción de un conjunto de valoraciones; (b) la clasificación de cada una de las valoraciones según una lista de términos afectivos; y (c) la discusión de los resultados con el cliente.

Las valoraciones y los términos afectivos se combinan en una matriz, en la cual las filas representan a las valoraciones y las columnas a los términos (Véase la Tabla 1 como ejemplo). A continuación explicaremos la metodología, ilustrándola posteriormente con un caso real.

\section{Formulación de las Valoraciones}

Las valoraciones son elicitadas por una serie de preguntas con final abierto. Las preguntas más importantes, presentadas en la Tabla 2, intentan sacar a relucir unidades de significado del pasado, presente y futuro que resultan importantes para la persona. Las preguntas se formulan de tal manera que invitan a los individuos a reflexionar sobre su situación vital, y a relatar fragmentos significativos de sus auto-narrativas. Los sujetos son libres de mencionar todas las preocupaciones que vean como más relevantes para ellos, y de interpretar las preguntas en el modo que deseen. Se les indica que han de expresar las valoraciones utilizando sus propias palabras de modo que las formulaciones de las mismas concuerden, en la medida de lo posible, con lo que pretendian que significaran. La forma típica que adopta una valoración es la de una frase, considerada como la unidad básica del texto (James, 1890). En esta frase los sujetos reúnen aquellos sucesos que sienten como elementos pertenecientes a una unidad de significado personal. No hay relación uno a uno entre pregunta y respuesta, y no se requieren respuestas rápidas. Cada pregunta conduce a más de una valoración, y los sujetos pueden mencionar tantas valoraciones cómo les lleguen a la mente. Al final de la entrevista se les pregunta si la misma contiene todas las experiencias que quisieran incluir en la investigación, de modo que si faltara alguna, puedan añadirla. En casos especiales, el clínico o terapeuta puede formular valoraciones adicionales y proponer al cliente que las incluya en el sistema de valoración (véase Hermans y Hermans-Jansen, 1995, p.64-67). Al final del procedimiento, el número de valoraciones puede variar enormemente, pero, en la mayoria de los casos, oscila entre 20 y 40 . Cada valoración es escrita por el entrevistador en tarjetas aparte $y$, en tal formato, quedan listas para la segunda parte de la investigación.

\section{Las Propiedades Afectivas de las Valoraciones}

En la segunda parte de la investigación, se proporciona al sujeto una lista estándar de términos afectivos. Centrándose en la primera valoración, los sujetos indican en una escala de 0 a 5 puntos el grado en el cual experimentan cada uno de los afectos de la lista en relación a la valoración $(0=\mathrm{Nada}$ en Absoluto, 1 = Un Poco, 2 = Algo, 3 = Bastante, 4 $=$ Mucho, y $5=$ Muchísimo). En esta fase de la investigación, los sujetos trabajan solos evaluando cada valoración con la misma lista de términos afectivos. Esto posibilita la comparación de las diferentes valoraciones en relación a sus perfiles afectivos. La lista de términos afectivos se muestra en la parte inferior de la Tabla 1. Esta lista proporciona 
Tabla 1.- Matriz Valoración x Afecto: Puntuaciones directas de la Auto-Investigación de un Sujeto.

\begin{tabular}{|c|c|c|c|c|c|c|c|c|c|c|c|c|c|c|c|c|c|}
\hline \multicolumn{18}{|c|}{ Términos Afectivos } \\
\hline No Valoración & 1 & 2 & 3 & 4 & 5 & 6 & 7 & 8 & 9 & 10 & 11 & 12 & 13 & 14 & 15 & \multicolumn{2}{|c|}{16} \\
\hline 1 & 1 & 1 & 1 & 5 & 1 & 1 & 5 & 5 & 5 & 4 & 1 & 1 & 5 & 3 & 2 & & 0 \\
\hline 2 & 0 & 0 & 0 & 5 & 0 & 0 & 5 & 0 & 5 & 0 & 0 & 0 & 5 & 0 & 5 & & 0 \\
\hline 3 & 1 & 0 & 1 & 5 & 0 & 0 & 5 & 5 & 4 & 5 & 0 & 1 & 5 & 0 & 4 & & 0 \\
\hline 4 & 0 & 0 & 0 & 4 & 0 & 0 & 0 & 0 & 5 & 0 & 0 & 0 & 4 & 0 & 4 & & 0 \\
\hline 5 & 5 & 1 & 4 & 2 & 1 & 4 & 4 & 5 & 1 & 4 & 3 & 4 & 1 & 4 & 1 & & 5 \\
\hline 6 & 0 & 0 & 4 & 4 & 1 & 0 & 3 & 1 & 5 & 0 & 0 & 0 & 2 & 0 & 4 & & 0 \\
\hline \multirow{2}{*}{$\begin{array}{l}\text { Sentimiento General } \\
\text { Sentimiento ideal }\end{array}$} & 1 & 0 & 1 & 4 & 0 & 1 & 4 & 4 & 4 & 1 & 1 & 1 & 5 & 1 & 4 & \multirow{2}{*}{\multicolumn{2}{|c|}{5}} \\
\hline & 4 & 5 & 4 & 0 & 4 & 4 & 4 & 5 & 0 & 4 & 4 & 3 & 0 & 2 & 1 & & \\
\hline \multicolumn{18}{|l|}{ Nota } \\
\hline \multicolumn{18}{|c|}{$\begin{array}{l}\text { Las filas representan las valoraciones y las columnas representan los términos afectivos utilizados para los indices } S, O, P, \text { y } N \text {, en los que } S= \\
\text { Afecto referido a la Auto-lmportancia; } O=\text { Afecto referido a la Relación con Otro; } P=\text { Afecto Positivo; y } N=\text { Afecto Negativo. Los términos de afecto son } \\
: 1=\text { Alegria }(P) ; 2=\text { Autoestima }(S) ; 3=\text { Felicidad }(P) ; 4=\text { Preocupación }(N) ; 5=\text { Fortaleza }(S) ; 6=\text { Disfrute }(P) ; 7=\text { Cuidado o Atención hacia los Otros } \\
\text { (O); } 8=\text { Amor }(O) ; 9=\text { Infelicidad }(N) ; 10=\text { Ternura }(O) ; 11=\text { Confianza en Uno Mismo }(S) ; 12=\text { Intimidad }(O) ; 13=P e s i m i s m o(N) ; 14=O \text { Orgullo }(S) ; 15= \\
\text { Decepción }(N) ; 16=\text { Paz Interior }(P) \text {. Los numeros del interior de la tabla son las puntuaciones directas asignadas por el cliente a cada afecto respecto } \\
\text { de cada valoración. Las puntuaciones provienen de la primera auto-investigación de Alexandra, la cliente que describiremos más adelante en este capitulo } \\
\text { en nuestro estudio de caso. }\end{array}$} \\
\hline
\end{tabular}


Tabla 2.- Preguntas del Método de Auto-Confrontación

Conjunto 1: El Pasado

Con estas preguntas se intenta guiarle en la revisión de uno o más aspectos de su vida que pueden haber sido de gran importancia para usted.

- ¿Ha habido algo de importancia capital en su vida pasada que todavia sigue ejerciendo una fuerte influencia sobre usted?

- ¿Hubo en el pasado alguna persona o personas, experiencia o circunstancias que influyeran enormemente en su vida y que afecten todavia de manera apreciable su existencia presente?

Conjunto 2: El Presente

Este conjunto de preguntas se compone nuevamente de dos preguntas que le llevarán, tras una cierta reflexión, a emitir una respuesta:

- ¿Hay algo en su existencia presente de importancia capital para usted o que ejerza una influencia significativa sobre usted?

- ¿Hay en su existencia presente alguna persona, personas, o circunstancias qué ejerzan una influencia significativa sobre usted?

\section{Conjunto 3: El Futuro}

Las siguientes preguntas le guiarán de nuevo hacia una respuesta:

- ¿Prevee usted la ocurrencia de algo que sea de gran importancia para usted, o que ejercerá una influencia capital en su vida futura?

- ¿Siente usted que cierta persona, personas o circunstancias ejercerán una influencia significativa sobre su vida futura?

- ¿Hay alguna meta futura u objetivo que usted espera jugará un importante papel en su vida?

Usted es libre de proyectarse tan lejos en el futuro como lo desee.

la cantidad máxima de información afectiva con el minimo de términos. Tras la estimación afectiva de las diferentes valoraciones, se calculan los indices que representan la estructura motivacional del sistema de valoraciones (para el uso clínico recomendamos una lista más amplia, véase Hermans y HermansJansen, 1995, p. 277).

1. El indice $S$ es la puntuación total de los cuatro términos afectivos que expresan AutoImportancia: números $2,5,11$, y 14 de la Tabla 1.

2. El indice $O$ es la puntuación total de los cuatro términos afectivos que expresan Relación y Unión con Otro: números 7, 8, 10, y 12 de la Tabla 1. Además, para cada valoración puede determinarse la diferencia S-O. Cuando la experiencia de Auto-Importancia es más fuerte que la experiencia de Relación con Otro, $\mathrm{S}>\mathrm{O}$. Cuando el sentimiento de Relación con Otro prevalece, O>S. Cuando ambos tipos de experiencia coexisten, $S=O$.
3. El índice $P$ es la puntuación total de cuatro términos de Afecto Positivo: números $1,3,6$, y 16 .

4. El índice $N$ es la puntuación total de cuatro términos de Afecto Negativo: números $4,9,13$, y 15. Para cada valoración puede determinarse de nuevo la diferencia $P-N$. Esto indica el grado de Bienestar que la persona experiencia en relación a la valoración especifica. El bienestar es positivo cuando $P>N$, negativo cuanto $N>P, y$ ambivalente cuando $P=N$. (Nótese que las puntuaciones para cada uno de los cuatro indices $\mathrm{S}, \mathrm{O}, \mathrm{P}$, y $\mathrm{N}$ oscilan de 0 a 20 para cada valoración).

5. El indice r representa la correspondencia entre los perfiles afectivos de dos valoraciones, calculada como la correlación entre dos filas cualesquiera de la matriz. Esta correlación indica la semejanza existente entre los significados afectivos de dos valoraciones, expresada mediante la forma que adoptan sus perfiles afectivos. La correlación muestra, a 
menudo, que las valoraciones referidas a sucesos diferentes entre si (p.ej., un comentario hecho en el pasado por un profesor y la experiencia en el presente de algún proyecto) tienen, aún con todo, perfiles afectivos muy similares en el mismo individuo.

El índice $r$ se utiliza también para la medir el alcance de la Generalización (Gen.) de una valoración dentro del sistema. Esto se hace preguntando a la persona, al final de la fase de construcción de las valoraciones: "¿Cómo te sientes en general estos dias?". Esta pregunta no exige una valoración específica sino que está diseñada para evaluar el "Sentimiento General". La persona responde directamente utilizando la misma lista de términos afectivos, como hizo para la caracterización de las valoraciones. Este patrón de puntuaciones se cumplimenta en una fila adicional en la matriz (véase la Tabla 1). La correlación momentoproducto entre el perfil afectivo perteneciente a una valoración especifica (cualquier fila de la matriz) y el perfil afectivo del "Sentimiento General" es una medida del grado de generalización de esta valoración. Cuanto más positiva sea la correlación, más se espera que tal valoración se generalice en el sistema. Por ejemplo, cuando un estudiante se preocupa mucho por sus estudios, una valoración como "Estoy pensando en mis estudios todo el tiempo" mostrará un patrón afectivo que correlacionará de forma elevada con el patrón afectivo perteneciente al "Sentimiento General". El perfil de puntuación del sentimiento general se puede utilizar también para evaluar el 'bienestar general'. Esto quedará indicado por la diferencia P-N en Sentimiento General.

De forma similar, el grado de Idealización (Id.) de cualquier valoración puede evaluarse calculando la correlación con el "Sentimiento Ideal". El Sentimiento Ideal es el perfil afectivo proporcionado como respuesta a la pregunta “¿Cómo te gustaría sentirte?”. Este perfil se cumplimenta en la última fila de la matriz. El indice de Idealización (Id.) es la correlación de cualquier valoración con el perfil característico del Sentimiento Ideal.

Por último, el índice $r$ puede utilizarse para comparar una valoración con cualquier otra. Una técnica especial, denominada Análisis de Modalidad, se utiliza para comparar una valoración particular con todas las demás. Para el Análisis de Modalidad, se toma una valoración concreta como punto central y se correlaciona con todas las demás valoraciones para buscar comunalidades subyacentes. (Para la fiabilidad y validez de los indices $S, O, P, N$, y $r$ véase Hermans, 1987a, p.166 y pp. 169171; para la combinación de investigaciones nomotéticas e idiográficas véase Hermans, 1988).

\section{La Discusión con el Sujeto}

La discusión de los resultados tiene lugar, normalmente, una semana después de la autoinvestigación. Durante esa semana, el entrevistador ha llevado a cabo un análisis cualitativo (estudiando la formulación de las valoraciones, con la adecuada atención a la relación que mantienen entre sí) y cuantitativo (cál-culo e interpretación de la combinación de los índices).

La discusión se dirige a la profundización de la auto-exploración de la persona, estimulada por un diálogo profundo con el entrevistador. Esta discusión se basa en el cuadro general proporcionado por el sistema como un todo integrado. En este sistema, las valoraciones divergentes se contraen a un momento en el tiempo de modo que nuevas relaciones, hasta ahora ocultas, pueden hacerse visibles. Esta visión global es muy característica de la auto-investigación. Mientras que las charlas cortas y las entrevistas normalmente están dispersas en el tiempo y, por lo tanto, poseen una cualidad momentánea, el cuadro global de una auto-investigación proporciona una perspectiva a "vista de pájaro" que permite una apreciación del sí mismo y de sus limites. Los sujetos ven qué valoran y qué no. Es más, se hacen conscientes de las lagunas presentes en su sistema de valoraciones. Esta confrontación con el contenido y la organización del sistema de valoración se beneficia, además, de la explicación de los motivos básicos. La persona puede ver en qué valoraciones se manifiestan $y$ en cuales no.

El propósito de la discusión no es sólo desarrollar insights, sino también, generar nuevas ideas, hacer planes. Esto no está confinado necesariamente a una sesión. Es incluso mejor proseguir con ello en sesiones sucesivas de modo que las experiencias cotidianas 
se puedan comparar con el contenido y la organización del sistema de valoraciones. Aquí se hace manifiesto que de la evaluación se pasa, gradualmente, al cambio, en tanto que una reflexión exhaustiva sobre la organización del sistema es el primer paso para la reorganización del mismo.

\section{La Segunda Auto-Investigación}

La segunda auto-investigación, que tiene lugar normalmente transcurridos algunos meses, consiste en las mismas fases que la primera. Sin embargo, hay una diferencia importante en la fase de construcción de las valoraciones. Esta vez, los sujetos no empiezan formulando valoraciones. En lugar de eso, son confrontados con las afirmaciones realizadas en la Investigación 1. El entrevistador y la persona, leen las preguntas de nuevo pero, después de cada pregunta, el entrevistador reproduce la afirmación que la persona consideró adecuada en la Investigación 1. Se indica a los sujetos que, al tomar cada valoración por separado, consideren si todavía están de acuerdo con su contenido, es decir, si darian la misma respuesta a las preguntas en su nueva situación. Si éste no fuera el caso, hay varias opciones disponibles: una vieja evaluación puede reformularse (Modificación); puede ser reemplazada por una nueva (Sustitución); puede descartarse (Eliminación); o puede añadirse una valoración nueva (Complementación). Este procedimiento garantiza que los sujetos tengan la libertad suficiente como para señalar las partes constantes y cambiantes del sistema de valoraciones.

\section{LA AUTO-INVESTIGACIÓN DE ALEXANDRA: EVALUACIÓN, CAMBIO, Y VALORACIÓN}

Alexandra, una mujer casada de 40 años con dos hijos adolescentes, contactó con un terapeuta durante un periodo en el cual estaba teniendo serias dificultades en su trabajo. En el colegio donde enseñaba se habia visto envuelta en un conflicto surgido entre la administración y sus compañeros. Alexandra era amiga de la pareja que dirigía el colegio y se convirtió, por lo tanto, en la "intermediaria" a la que tanto la administración como sus colegas acudian para resolver los conflictos. Cuando al final la administración abandonó la escuela, su situación fué de mal en peor, porque ahora no tenia ningún papel (atenuante) que desempeñar. Además, tenia que tomar la difícil decisión de permanecer en una situación laboral en la que carecia de apoyo o dejar su trabajo. Experiencias del pasado sin resolver se re-actualizaron por los problemas presentes en su trabajo, y abocaron a esta mujer a una crisis.

Alexandra relató lo siguiente acerca de sus antecedentes familiares. Creció en una familia holandesa de clase media. Era la mayor de cinco hijos. Describía a su padre como una persona muy autoritaria que ejercia un control absoluto sobre la situación en casa; mantenia una fuerte actitud normativa hacia los roles padre-hijo y los roles de género; requeria obediencia inmediata por parte de sus hijos y los ponía bajo "arresto domiciliario" cuando rompian alguna de sus estrictas reglas. La madre de Alexandra adoptó un papel muy subordinado en la familia; estaba enferma con frecuencia y padecía de asma. Como resultado de la debilidad fisica de su madre, Alexandra se convirtió en una "segunda madre" para la familia. Su padre le otorgaba responsabilidades sociales especiales en relación a sus hermanos más jóvenes y a la familia como un todo, justificándolo frecuentemente con la observación "Tú eres la mayor y, por lo tanto, la más juiciosa". Las relaciones familiares se veian complicadas, además, por el infeliz matrimonio de los padres. Alexandra se describia a si misma como viviendo con el constante miedo de que sus padres se separaran y, como hija mayor, trataba continuamente de "mantener la paz" en la familia. Su ansiedad no sólo concernía a la separación de sus padres sino a la ruptura familiar $y$ al peligro de tener que tomar partido: ella sentia que tenia que evitar tomar partido porque el hacerlo, o incluso el dar la impresión de hacerlo, conduciría a la separación de sus padres y a la ruptura de la familia como un todo.

Alexandra llevó a cabo dos auto-investigaciones, con nueve meses de intervalo entre ambas. Además, se realizó una valoración a la mitad del proceso para examinar la dirección del proceso de cambio iniciado 


\section{La Primera Auto-Investigación}

En la primera sesión con el psicoterapeuta, se discutieron la naturaleza y objetivos del procedimiento de auto-confrontación, y se hicieron explicitas las expectativas del cliente y del terapeuta. En la segunda sesión, se formularon las valoraciones y se asociaron con el conjunto de términos afectivos estandarizado descrito anteriormente. Para ilustrar el proceso presentamos en la Tabla 3 seis valoraciones del sistema de Alexandra, dos referidas a su pasado ( 1 y 2), dos a su presente (3 y 4$), y$ dos a su futuro $(5$ y 6$)$.

Al comparar las valoraciones referidas a su pasado con aquellas pertenecientes a su situación presente, vemos una re-actualización de problemas del pasado. Se sugirió que el problema de ser una "intermediaria" (véase la valoración $n^{\circ} 4$ en la Tabla 3) estaba confirmando su experiencia pasada de encontrarse "entre dos bandos" (véase la valoración $n^{\circ} 2$ en la Tabla 3).

Una característica sobresaliente del sistema de Alexandra es el papel dominante de la experiencia de Amor Insatisfecho (véanse las valoraciones $n^{\circ s} 1$ y 3 . Para una discusión teórica véase el artículo anterior). La investigación empirica sobre esta experiencia (Hermans y Van Gilst, 1991; Hermans y Hermans-Jansen, 1995) ha mostrado que ésta quedaba expresada por el siguiente patrón afectivo: $S$ bajo, $\mathrm{O}$ alto, $\mathrm{P}$ bajo, y $\mathrm{N}$ alto. $\mathrm{Ob}$ servando la estructura formal de esta experiencia pueden identificarse dos componentes: (a) hay una orientación amorosa hacia otra persona $u$ objeto (Amor, indicado por la diferencia $O>S$ ), y (b) hay un obstáculo o límite que hace a esta persona $u$ objeto inalcanzable (Insatisfecho, indicado por la diferencia N>P). En términos teóricos esta estructura representa la base latente de una amplia variedad de valoraciones personales en el nivel manifiesto. Dependiendo de la historia del individuo, la experiencia de amor insatisfecho tiene varias manifestaciones: la muerte de un amigo querido, un amor imposible, un emigrante nostálgico y anhelante de su país nativo, la anticipación de una despedida, un Fausto nostálgico de la juventud, o sentirse cercano a alguien $y$, al mismo tiempo, incapaz de expresarle ternura y simpatía.
En el caso de Alexandra vemos que los perfiles afectivos asociados con las valoraciones 1 y 3 tienden hacia un patrón afectivo de Amor Insatisfecho. Este perfil afectivo tiene una generalización relativamente acusada en el sistema como conjunto. Una clara indicación de esto es el patrón afectivo del "Sentimiento General" que también se asocia con bajo $S$, alto $O$, bajo $P, y$ alto $N$. Nótese también el alto nivel de Generalización (Gen.) de las valoraciones 1 y 3 (Tabla 3). La dominancia del tema de Amor Insatisfecho en el componente afectivo de las valoraciones de Alexandra indica que se siente marcadamente dependiente de los otros.

\section{La Reorganización del Sistema de Valoraciones}

Aunque las valoraciones indicativas de Amor Insatisfecho son, desde un punto de vista diagnóstico, influyentes en el sistema de valoraciones, no jugaban el papel primario en la reorganización del sistema de Alexandra. La valoración que desempeñaba el papel crucial en tal reorganización del sistema como un todo era la $n^{\circ} 6$ : "Me gustaria mostrarme exteriormente como la persona que soy interiormente, pero nunca se lo he dejado ver a nadie (por encima de todo mostrarme más fuerte $y$ decidida)". Alexandra explicó que ella realmente veía comportamientos fuertes y decididos en otras personas, pero no en sí misma. Por ejemplo, relató como su hermano pequeño podía replicar a su padre y añadió "Yo nunca me atreveria a hacerlo". Dado que no se atrevía a ser tal persona, asociaba su valoración $\left(n^{\circ} 6\right)$ con un nivel muy bajo de AutoImportancia y con un alto nivel de Afecto $\mathrm{Ne}$ gativo. Al mismo tiempo, Alexandra estaba fascinada por la fuerza y la firmeza, y sentia que una persona con tales características se encontraba en alguna parte "profundamente dentro" de si misma. El psicoterapeuta sospechó que la valoración anterior podria, quizá, funcionar como una "puerta al cambio" y sugirió a Alexandra que se concentrara en la significación afectiva de esa valoración en la sesión siguiente. Esto se realizó tomando la valoración $n^{\circ} 6$ como la central en un análisis de modalidad, y se la correlacionó con todas las otras valoraciones del sistema (véase la 
columna final de la Tabla 3 para los resultados). Las valoraciones que mostraban la más alta correlación con la $n^{\circ} 6$ fueron examinadas en función de su tema común. Alexandra proporcionó las siguientes interpretaciones (de las correlaciones más altas a las más bajas):

$N^{\circ} 6 \times n^{\circ} 2(r=.92)$ : "Cuando se me pide hacer algo y pienso que no soy lo suficientemente fuerte, me cierro del todo."

$N^{\circ} 6 \times n^{\circ} 4$ ( $r=.85$ ): "Una parte (la administración) me sobreestimaba y la otra parte (los compañeros) me subestimaba... pero yo no me mostré como era en realidad... Cuando podría haberme manifestado yo misma más..."

Después de interpretar cierto número de otros pares de valoraciones de manera similar, el terapeuta invitó a Alexandra a resumir qué veía como tema común subyacente en todas sus interpretaciones. Tras varios intentos, Alexandra descubrió lo siguiente: "Nunca he tenido que luchar por algo por mi misma... casi todo ha sido siempre dispuesto para mi por otros." En otras palabras, Alexandra no habia tenido nunca la ocasión, o no la había aprovechado, de obtener un grado mínimo de Auto-Importancia como persona independiente (véase también el bajo nivel de afecto $S$ en la Tabla 3). Desde una perspectiva teórica, podemos concluir que este resumen puede ser considerado como un tema central en la auto-narrativa de Alexandra, y que este tema habia sido hecho explicito al tomar en consideración la base latente motivacional del sistema de valoraciones.

Tras el resumen era hora de una concentrada auto-reflexión y discusión acerca de la influencia generalizada del tema base. Alexandra se fue a casa con el conocimiento de que en la auto-investigación se habia enfrentado a un tema esencial en su vida. Era lógico pararse a mirar sólo lo que ocurre durante el periodo justo tras la minuciosa auto-investigación. Es decir, Alexandra fue invitada a observar, desde la perspectiva del tema base, cómo era vivida su vida, y qué ocurría entre ella misma y su ambiente.

Como muchos psicoterapeutas han observado, la mejor estrategia para animar al cambio no es subrayar la necesidad o urgencia del cambio sino, más bien, concentrarse en la naturaleza de las propias experiencias personales. Mucha gente, impacientes por el cam- bio y afanándose de forma consciente en conseguirlo, paradójicamente fracasan debido a los crecientes sentimientos de estrés y a las prontas decepciones que bloquean el proceso más que promoverlo. El proceso de cambio requiere una auto-reflexión y concentración serias, permaneciendo quieto por un momento para mirar y llegar a conocer el/los tema/s fundamentos del propio sistema de valoraciones, incluso cuando éstos son dolorosos.

Sorprendentemente, la persona bajo tal condición de auto-reflexión concentrada empieza, a menudo de forma espontánea, a tomar ciertas iniciativas, a explorar alternativas y a probar con unos pocos ensayos y errores. Estos pequeños cambios son sondeados con frecuencia sabiendo que "esto puede ser diferente". Es decir, el sistema de valoraciones, tal y como está actualmente formulado, no es la última palabra, no es "perfil de personalidad último", no es un yo fijo. Más bien, el sistema de valoraciones del individuo es un comienzo $y$, en el contexto de una relación de apoyo con el terapeuta, el cliente empieza el proceso de cambio, empieza a relatar de nuevo partes de su auto-narrativa bajo una luz diferente. Esta perspectiva cambiante se manifiesta especialmente cuando algunos sucesos nuevos son añadidos por el individuo al sistema de valoraciones. Esta alteración del sistema indica, a su vez, la capacidad de auto-organización de la persona. (Para un procedimiento más detallado y sistemático para estimular el cambio en el sistema de valoraciones, véase el ciclo Atender-Crear-Anclar, Hermans y HermansJansen, 1995, p. 47-54).

\section{La Evaluación Intermedia: Comprobando la Dirección del Cambio}

De acuerdo con la noción de cambio autoinstigado, Alexandra empezó a tomar nuevas iniciativas que eran discutidas posteriormente con el terapeuta cada semana, en estrecha correspondencia con el sistema de valoraciones de la primera auto-investigación. Estas "iniciativas" se relacionaban con el "vacio" que se habia evaluado en su sistema: el bajo nivel de Auto-Importancia en el mismo. Algunas de estas iniciativas se preparaban en las sesiones con el terapeuta, mientras que otras ocurrian espontáneamente y eran discutidas 
Tabla 3.- Valoraciones de Alexandra. Sentimiento General, Sentimiento ldeal y Puntuaciones en los indices Afectivos en el Momento 1.

\begin{tabular}{|c|c|c|c|c|c|c|c|}
\hline Valoración & S & O & $P$ & $\mathbf{N}$ & Gen. & Id. & $n^{\circ} 6$ \\
\hline $\begin{array}{l}\text { 1. Siempre he tenido miedo de perder a la gente (el matrimonio de mis padres no era } \\
\text { bueno: siempre habia tensión); siempre que puedc trato de evitar situaciones de conflicto }\end{array}$ & 6 & 15 & 3 & 17 & .83 & -.54 & .62 \\
\hline $\begin{array}{l}\text { y yo no sabia qué hacer (estar entre dos fuegos) } \\
\text { 3. Pienso que es muy malo que mis hijos no puedan resultar indemnes, pues ellos también }\end{array}$ & 0 & 5 & 0 & 20 & .86 & -.78 & .92 \\
\hline sufren el tener una madre problemática & 0 & 16 & 2 & 18 & .86 &. .48 & .66 \\
\hline 4. En el conflicto del colegio me siento como un amortiguador entre dos grupos enfrentados & 0 & 0 & 0 & 17 & .74 & -.91 & .85 \\
\hline $\begin{array}{l}\text { 5. Espero poder tranquilizarme en el futuro (respecto a mis sentimientos) } \\
\text { 6. Me gustaria mostrarme exteriormente como la persona que soy interiormente, pero nunca }\end{array}$ & 9 & 17 & 18 & 5 & -.30 & .58 & -.55 \\
\hline se to he dejado ver a nadie (por encima de todo mostrarme más fuerte y decidida) & 1 & 4 & 1 & 15 & .79 &. .73 & - \\
\hline Sentimiento General & 2 & 10 & 3 & 17 & - &. .67 & .79 \\
\hline Sentimiento Ideal & 15 & 16 & 17 & 1 & -.67 & 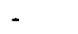 & -.73 \\
\hline
\end{tabular}

Tabla 4.- Valoraciones del Cambio de Alexandra, Sentimiento General, Sesiones con el Psicoterapeuta, y Auto-Investigación durante la Evaluación Intermedia

\begin{tabular}{|c|c|c|c|c|c|}
\hline Valoración & $S$ & 0 & $P$ & N & Gen. \\
\hline 1. He ganado más seguridad en mi misma; me atrevo a hacer más cosas & 8 & 12 & 10 & 5 & .73 \\
\hline la administración y de mi madre) & 6 & 11 & 10 & 4 & .75 \\
\hline $\begin{array}{l}\text { 3. Hago más lo que yo quiero y no me preocupo tanto por lo que otros esperan; } \\
\text { lo que otros piensen que yo debería hacer no me preocupa } \\
\text { Sesiones con el psicoterapeuta. } 117111.19\end{array}$ & 9 & 91 & 2 & 1 & .65 \\
\hline $\begin{array}{l}\text { Mi Auto-investigación } \\
\text { Sentimiento General }\end{array}$ & $\begin{array}{l}8 \\
6\end{array}$ & $\begin{array}{l}14 \\
12\end{array}$ & $\begin{array}{l}11 \\
9\end{array}$ & $\begin{array}{l}3 \\
3\end{array}$ & 69 \\
\hline
\end{tabular}


más tarde. Por ejemplo, hubo un malentendido en el colegio de su hija y decidió escribir una carta para aclarar las cosas, aunque previamente hubiera dejado esta tarea para su marido; en una ocasión oyó que una vecina la habia acusado de una posible falta de honradez, así que contactó con ella para que le explicara porqué, a pesar de que solia evitar el conflicto a toda costa; de forma similar, en una conversación con el representante de cierta organización que le pedia dar una descripción de si misma, ella mencionó algunos "puntos fuertes" acerca de si, aunque antes hubiera considerado eso como estar "alardeando".

Durante los tres meses que siguieron a la primera auto-investigación, el terapeuta propuso llevar a cabo una auto-investigación limitada para comprobar la dirección y naturaleza del cambio que estaba teniendo lugar. Esto se llevó a cabo preguntando simplemente a Alexandra cuáles pensaba que habian sido los principales cambios desde la investigación inicial. Estos cambios se formularon en forma de valoraciones y este pequeño conjunto de valoraciones fué puntuado con la lista estándar de términos afectivos. Con fines evaluativos, las sesiones con el psicoterapeuta, la propia auto-investigación, y el Sentimiento General fueron también valorados con el mismo conjunto de términos afectivos.

En la Tabla 4 vemos lo que Alexandra percibe que son los cambios principales, formulados como tres valoraciones ( $n^{\circ s} 1,2$, y 3 ). Un rasgo relevante de estas valoraciones de cambio es su naturaleza comparativa. El presente es percibido ahora como diferente del pasado. Aunque el contenido de las formulaciones sugiere un aumento en la Auto-Importancia, los indices afectivos muestran que el afecto $O$ todavia tiende a ser más elevado que el afecto $S$. Esto también es cierto para el Sentimiento General, que es ahora más positivo, pero aún muestra un nivel bastante bajo de afecto $S$.

Una comparación de las estimaciones de Alexandra sobre las sesiones con el psicoterapeuta y su propia auto-investigación muestran que ambas son experimentadas como positivas; estas actividades han encontrado de forma clara una "entrada" en el sistema de valoración. Las sesiones de psicoterapia tienden a tener más afecto $S$ que afecto $O$, su- giriendo que pueden ser el precursor de posteriores cambios de valoraciones y del fortalecimiento del motivo $\mathrm{S}$.

Para resumir, esta evaluación intermedia muestra los comienzos del cambio en la dirección deseada, a saber, las características de la persona de mentalidad fuerte y decidida representadas en la valoración $n^{\circ} 6$ (Tabla 3 ). Empieza a perfilarse el incremento en la AutoImportancia, aunque la mayoria de las valoraciones de cambio en si mismas aún muestran una predominancia del afecto referente al motivo de Contacto con Otro.

\section{La Segunda Auto-investigación: Reorganización}

Nueve meses después de la auto-investigación inicial, cliente y psicoterapeuta sintieron que había cambiado tanto la visión de Alexandra sobre si misma y el mundo que decidieron llevar a cabo una segunda auto-investigación. Los resultados se presentan en la Tabla 5, y los principales cambios se resumen a continuación.

En la segunda investigación vemos una ruptura en las valoraciones de Auto-Importancia del sisterna. Las valoraciones 1 y 2 en el Momento 1 han sido modificadas, expresando más Confianza en Uno Mismo ( $\left.n^{\circ} 1\right)$ y más Espíritu de Lucha ( $\left.n^{\circ} 2\right)$. Esto se evidencia también en los altos niveles de afecto $S$. Las otras valoraciones ( $n^{\text {os }} 4,5$, y 6 ) muestran claramente niveles más altos de afecto $S$ que de afecto $O$. Esto sugiere que Alexandra se siente capaz de anteponer sus intereses a los de los demás cuando es necesario (nótese que este tipo de valoración, $\mathrm{S}>\mathrm{O}$, estaba completamente ausente en el Momento 1). Esta reorganización implica que ha resuelto gran parte de sus problemas personales mediante el descubrimiento en si misma de fuerza, y este aumento en la Fortaleza y en la Confianza en si Misma le han permitido decidirse por el abandono de su trabajo $\left(n^{\circ} 4\right)$. Esto elimina una carga importante en el sistema de valoraciones y también los sentimientos que estaban reactualizando otras experiencias estresantes de su pasado.

La valoración que en el Momento 1 se referia a su deseo de ser fuerte en su exterior $\left(n^{\circ} 6\right)$ habia adquirido proporciones más rea- 
Tabla 5.- Valoraciones de Alexandra, Sentimiento General, Sentimiento Ideal y Puntuaciones en los Indices Afectivos en el Momento 2.

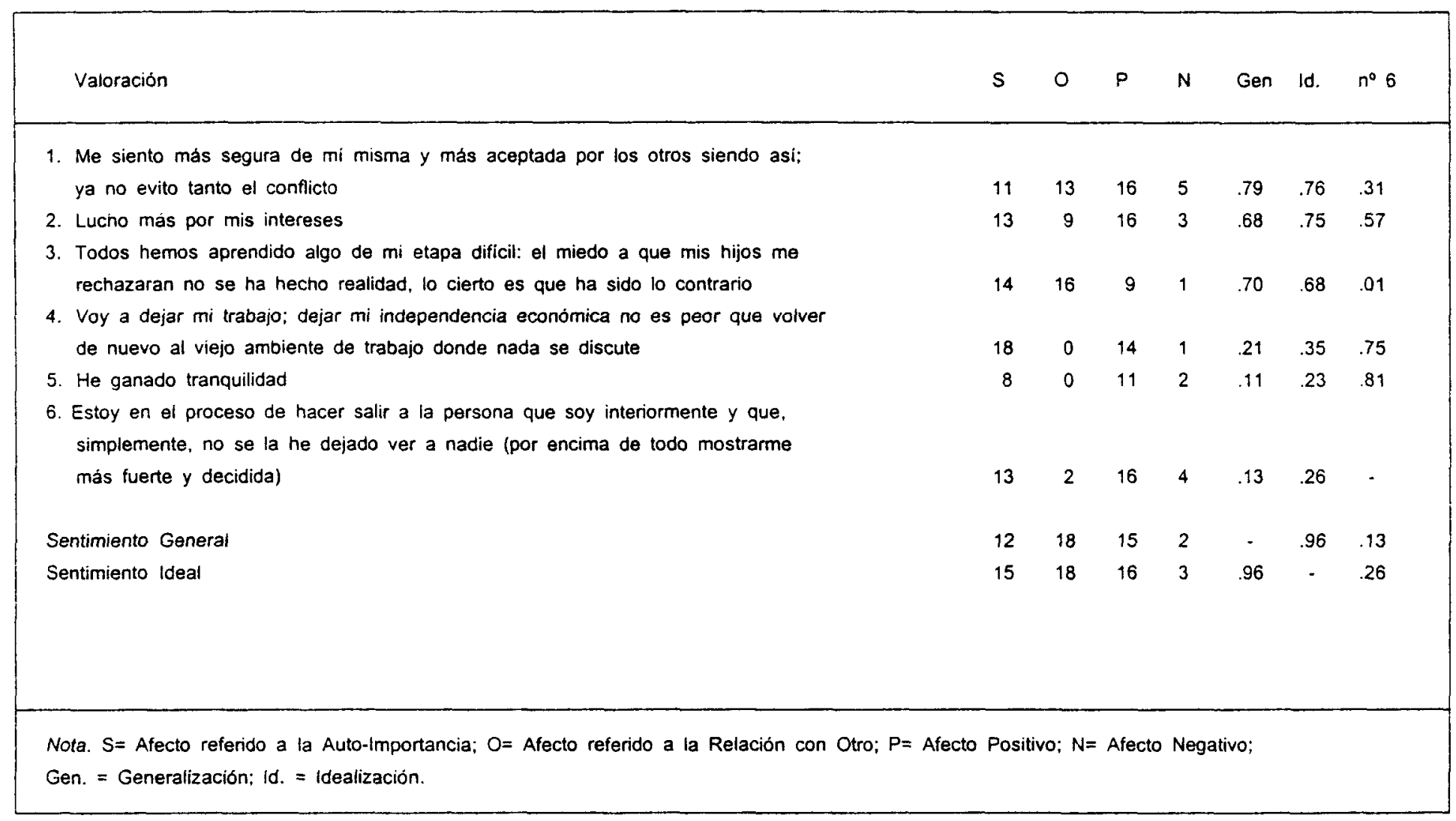


listas en el Momento 2. Cuando esta valoración se correlaciona con todas las demás (véase la columna final de la Tabla 5), se hallan correlaciones altas con valoraciones del tipo $S>0$ principalmente (nos 2, 4, y 5). Esto indica que la fuerza potencial insinuada por la valoración $n^{\circ} 6$ ha sido integrada dentro del sistema de valoraciones. (Nótese que el Sentimiento General tiene todavia una $O$ más alta que la $S$, sugiriendo que el elevado nivel de afecto $S$ no se ha generalizado todavia en el sistema).

\section{RESUMEN DEL CASO PRESENTADO}

El caso de Alexandra puede ser visto como una auto-narrativa que originalmente mostraba una omisión notable. En parte sobre la base de los valores colectivos (su educación como mujer), y en parte sobre la base de su historia individual (hija mayor en una familia con la madre enferma, el infeliz matrimonio de sus padres), Alexandra desarrolló la identidad de "intermediaria", una persona que está constantemente cuidando de los otros y esforzándose por mantener todas y cada una de sus relaciones con ellos. En consecuencia, no tenia oportunidad de, o simplemente no se le permitia, desarrollar un yo independiente. No obstante, Alexandra podia imaginarse claramente a si misma como una persona fuerte, decidida, y asertiva, que estaba escondida "profundamente dentro" de ella pero que no era, desde su punto de vista, parte de su conducta actual. Sin embargo, haciendo uso de las posibilidades que sentia en si misma y del apoyo del psicoterapeuta, salió finalmente de su crisis. Es más, su caso demuestra que una transición, e incluso una crisis, implican peligros $y$ oportunidades: Alexandra rellenó la omisión original de su sistema de valoraciones con la fortaleza, que habia estado escondida e incluso prohibida hasta entonces. Esto resultó en un sistema de valoraciones más integrado y flexible, y también le permitió detener el ciclo recurrente de preocupaciones impuestas y normativas.

Este caso ha sido presentado para demostrar como operan la evaluación, el cambio y los aspectos valorativos del procedimiento de auto-confrontación. Por supuesto que éste es un ejemplo que muestra como el método funciona en un caso óptimo, expresamente seleccionado. En este ejemplo se combinaron las tres funciones necesarias para llevar a cabo una transición suave entre la evaluación y el cambio. Sin embargo, éste no es siempre el caso y la transición depende en gran medida de las capacidades (las que quedan) de la persona para reorganizar su sistema de valoraciones. Un cliente en un estado depresivo, por ejemplo, caracterizado por el desamparo y la desesperanza, se siente con frecuencia incapaz de tomar las más mínimas iniciativas, e incluso cuando tal iniciativa es llevada a cabo puede ser absorbida rápidamente por los sentimientos dominantes y generalizados de desesperanza o apatía. En tal caso puede ocurrir que no haya una transición espontánea de la evaluación al cambio. Puede necesitarse un periodo más largo para llegar al punto donde puede intentarse la reorganización, y la paciencia, apoyo, y compromiso del psicoterapeuta, utilizando un abanico más amplio de técnicas, que sólo las descritas en este artículo, puede resultar crucial.

\section{ÁREAS PROBLEMA Y CAMPOS DE APLICACIÓN}

La teoría de valoración y el método de auto-confrontación se han diseñado para hacer contribuciones a la psicología considerada como un campo integrador. Esto significa que las contribuciones van a llegar a una variedad de subdisciplinas psicológicas. Uno de los objetivos es transcender la tradicional separación existente entre el científico y el profesional (véase Hoshmand y Polkinghorne, 1992). Esto se lleva a cabo mediante el uso del método, no sólo como un instrumento de investigación, sino también como un procedimiento que puede ser utilizado en la práctica para ayudar a las personas, consideradas como co-investigadores del psicólogo (Hermans y Bonarius, 1991), en su auto-organización y reorganización. Con estos propósitos en mente, teoria y métodos se han aplicado a una diversidad de tópicos psicológicos. A continuación mencionamos brevemente un conjunto representativo de estas contribuciones.

La teoria y el método han sido aplicados a problemas de identidad con un énfasis especial en la reorganización del sistema de 
valoraciones desde la perspectiva de los motivos básicos (Hermans, 1987b). Recurriendo al trabajo de Becker (1971) y Frankl (1973), se ha estudiado el significado de la vida como un proceso organizado (Hermans, 1989). En esta investigación los clientes expresan su experiencia subjetiva del significado de la vida en términos de un perfil afectivo que podria ser comparado con los perfiles afectivos de todas las demás valoraciones del sistema. Como resultado, pueden identificarse las valoraciones que hayan tenido más impacto en la forma en la que una persona da sentido a su vida como un todo.

Se ha realizado una amplia investigación sobre alteraciones clinicas, principalmente en depresiones, problemas psicosomáticos y fenómenos disociativos, en estrecha relación con las defensas psicológicas (Hermans y Hermans-Jansen, 1995). En un estudio del fenómeno de la promiscuidad, se ha desarrollado una técnica especial para subdividir una valoración particular en distintas partes. Estas partes son examinadas como (sub)valoraciones separadas, de modo que pueden evaluarse los aspectos suprimidos de una experiencia particular (Hermans y Hermans-Jansen, 1992). También se diseñó un procedimiento específico para el estudio de los contrarios en las valoraciones personales y su cambio desde una perspectiva jungiana (Hermans, 1993). Recientemente, se han traducido los constructos de Kelly (1955) a un nivel teórico como personajes con voz, dialogalmente relacionados en forma de protagonista y antagonista (Hermans, 1996).

En el terreno de la psicología educativa, Beem y Brugman (1986) desarrollaron y evaluaron un procedimiento especial para enseñar en el aula valoraciones a alumnos de entre 10 y 15 años. Poulie (1991) desarrolló un método de entrenamiento para reducir el miedo al fracaso y estudió los cambios en el contenido y en la estructura de las valoraciones de alumnos de entre 13 y 15 años. Sandfort (1984) desarrolló una versión adaptada del método de auto-confrontación e investigó las experiencias de chicos de entre 10 y 16 años implicados en relaciones pedofilicas.

Desde el punto de vista del desarrollo, Hermans (1992) demostró cómo cambiaban las valoraciones morales para una persona que relató y volvió a relatar su auto-narrativa durante un periodo de cambios radicales en su vida personal y profesional. Se demostró que las valoraciones morales cambiaban en estrecha correspondencia con cambios en las otras valoraciones del sistema. Hallazgos similares han sido aportados por Hermans y Van Loon (1991) y Van Loon (1992), los cuales exploraron el significado de los simbolos (p. ej. el árbol o el camino), encontrando que tales símbolos, como estímulos multi-interpretables, recibian significados enraizados en el sistema de valoraciones presente en ese momento. Cuando el sistema de valoraciones cambiaba, el significado del símbolo cambiaba a su vez.

El interés en los fenómenos colectivos instigó, asimismo, a Hermans y Oles (1994) a estudiar el papel de los valores en una sociedad rápidamente cambiante (Polonia en el periodo 1988-1991). Se encontró que los valores de Spranger (social, estético, religioso, teórico, y económico) evocaban valoraciones personales con más afecto positivo que negativo. Se halló una excepción para los valores politicos, los cuales estaban asociados con valoraciones que tenian más afecto negativo que positivo.

En suma, la experiencia con la teoria de valoración y el método de auto-confrontación en diversos campos sugiere que el estudio continuado del si mismo como fenómeno altamente complejo y dinámico, puede beneficiarse de una metodologia idiográfica basada en la teoria.

\section{REFERENCIAS BIBLIOGRÁFICAS}

Becker, E. (1971). The birth and the death of meaning: An interdisciplinary perspective on the problem of man. New York: The Free Press.

Beem, A.L. y Brugman, D. (1986). The effects of values development lessons on pupils' wellbeing, pleasure in school, mutual relationships and on pupils' valuational behavior during classroom dialogues. Theory and Research in Social Education, 14, 97-112.

Frankl, V.E. (1973). Psychotherapy and existentialism: Selected papers on logotherapy. Harmondsworth, Middlesex, England: Penguin Books. 
Hermans, H.J.M. (1987a). Self as organized system of valuations: toward a dialogue with the person. Journal of Counseling Psychology, 34, 10-19.

Hermans, H.J.M. (1987b). The dream in the process of valuation: A method of interpretation. Journal of Personality and Social Psychology, 53, 163175.

Hermans, H.J.M. (1988). On the integration of idiographic and nomothetic research method in the study of personal meaning. Journal of Personality, 56, 785-812.

Hermans, H.J.M. (1989). The meaning of life as an organized process. Psychotherapy, 26 (1), 1122.

Hermans, H.J.M. (1992). Telling and retelling one's self-narrative: A contextual approach to life-span development. Human Development, 35, 361375.

Hermans, H.J.M. (1993). Moving opposites in the self: A Heraclitean approach. Journal of Analytical Psychology, 38, 437-462.

Hermans, H.J.M. (1996). Opposites in a dialogical self: Constructs as characters. The Journal of Constructivist Psychology, 9, 1.26.

Hermans, H.J.M. y Bonarius, H. (1991). The person as co-investigator in personality research. European Journal of Personality, Vol. 5, 199216.

Hermans, H.J.M. y Hermans-Jansen, E. (1992). The personal valuation of promiscuity: A method of investigation. The Psychochotherapy Patient, Vol. 8(1-2), 121-140.

Herrmans, H.J.M. y Hermans-Jansen, E. (1995). Self-Narratives: The Construction of Meaning in
Psychotherapy. New York. Guilford Press.

Hemrans, H.J.M. y Oles, P. (1994). The personal meaning of values in a rapidly changing society. Journal of Social Psychology, 134, 569579.

Hermans, H.J.M. y Van Gilst, W. (1991). Selfnarrative and collective myth: An analysis of the Narcissus story. Canadian Journal of Behavioral Science, 23, 423-440.

Hermans, H.J.M. y Van Loon, R.J.P. (1991). The personal meaning of symbols. Journal of Religion and Health, 30, 24i-261.

Hoshmand, L.T. y Polkinghorne, D.E. (1992). Redefining the science-practice relationship and professional training. Amercian Psychologist, 47, 55-66.

James, W. (1890). The Principles of Psychology (Vol. 1). London: Macmillan.

Kelly, G.A. (1955). The psychology of personal constructs. New York. Norton.

Poulie, M.F. (1991). Meer licht op faalangst: De waardering van het individu [More light on fear of failure: The valuation of individual]. Dissertation. Nijmegen: University of Nijmegen.

Sandfort, Th. G.M. (1984). Sex in pedophiliac relationships: An empirical investigation among a nonrepresentative group of boys. The Journal of Sex Research, 20, 123-142.

Vanm Loon, R.J.P. (1992). Way symbolism in personal live, ilustrated and explained from a taoïst perspective. In P. Mischel (Ed.). Sumbolik von Weg und Reise. Schriften zur Symbolforshung (Vol. 8, pp. 299-236). Bern: Peter Lang. 